\title{
Viver próximo à saúde em Belo Horizonte
}

\author{
Living near health at Belo Horizonte
}

Renato Cesar Ferreira de Souza

Veneza Berenice de Oliveira

Doralice Barros Pereira

Heloisa Soares de Moura Costa

Waleska Teixeira Caiaffa

\begin{abstract}
Resumo
A definição de unidades espaciais como locus preferencial na prestação do cuidado em saúde é desafiadora quando se buscam modelos assistenciais que reduzam as iniquidades de acesso aos serviços de saúde e os humanizem, integrando dados demográficos, socioeconômicos, culturais e ambientais, configurando localmente os determinantes sociais da saúde. Este estudo introduz uma análise da localização das Unidades Básicas de Saúde (UBS) nos distritos sanitários de Belo Horizonte, comparando as divisões administrativas adotadas com as obtidas pelo teste cartográfico de influência de acesso às UBS. Demonstra-se a potencialidade de investigações futuras sobre o território da cidade na busca de melhores localizações e acesso às UBS, acenando para o enriquecimento do debate sobre os rumos da saúde urbana no Brasil.
\end{abstract}

Palavras-chave: análise espacial; espaço urbano; saúde urbana; acesso aos serviços de saúde; sistema único de saúde.

\begin{abstract}
TThe definition of spatial units as the preferential locus for healthcare provision is a challenging task when we seek healthcare models that humanize the health services and reduce inequities concerning access to them, integrating demographic, socioeconomic, cultural and environmental data, and configuring the social determinants of health at a local level. This study analyzes the location of Primary Care Units (PCU) in the Health Districts of the city of Belo Horizonte, and compares the adopted administrative divisions with those obtained by mapping the influence of shorter distances to the PCU. It demonstrates the potential that further research on the city's territory has in the search for better locations and access to the $P C U$, enriching the debate on the future of Urban Health in Brazil.
\end{abstract}

Keywords: spatial analysis; urban space; urban health; access to healthcare; Brazil's National Healthcare System. 


\section{Introdução}

O Sistema Único de Saúde do Brasil (SUS) é o maior sistema de saúde do mundo com características de universalidade, equidade e gratuidade. Desde sua criação, em 1988, um de seus maiores desafios é garantir o acesso de toda a população aos serviços de saúde com qualidade. Entretanto, ainda há uma realidade desigual e excludente significativa na distribuição desses serviços no País. Tenta-se, então, amenizar esse problema priorizando uma rede de Atenção Primária à Saúde (APS) em nível territorial, seja municipal ou por regiões de saúde, considerando-a como uma melhor porta de entrada para o SUS. As relações do território com vistas à organização dos serviços do SUS foram definidas pelo Decreto 7508/2011, no qual também se renova a importância da APS conforme as recomendações do Relatório Mundial de Saúde de 2008 (OMS, 2008).

Em Belo Horizonte, o mapeamento da distribuição de recursos humanos, de ações e de serviços de saúde ofertados pelo SUS passou a ser feito, colocando a estrutura do espaço público e suas dimensões físicas como integradoras, com outros aspectos ambientais e de indicadores de saúde, para nortear a priorização das implementações (Pitchon, 2013). Por essa razão, apresenta-se como cidade de interesse na avaliação da configuração de sua estrutura espacial de UBS. 0 critério do número de pessoas residentes numa determinada localidade foi utilizado na especificação da menor unidade espacial de atuação das equipes de saúde da família.

Na década de 1990, a Secretaria Municipal de Saúde de Belo Horizonte inovou ao implementar um modelo de APS que conjugava o critério estabelecido pelo SUS com balizamento do Índice de Vulnerabilidade Social (IVS) na definição das áreas prioritárias de atuação e do número de equipes de saúde da família para cada UBS. A cidade foi dividida em nove regiões administrativas, os Distritos Sanitários, onde está localizado um número diferenciado de UBS. Esses esforços se direcionaram à oferta de serviços de qualidade, com maior equidade, fácil acesso e em tempo adequado ao atendimento das necessidades de uma porção maior da população. Simultaneamente, visavam fortalecer a atenção aos ciclos da vida, com temáticas diversas, enfatizando-se, por exemplo, as áreas e as populações de maior vulnerabilidade. Esses aspectos conferiram extrema importância à discussão da localização das unidades e serviços da APS no espaço urbano, exigindo a problematização de metodologias para a sua avaliação e implementação.

\section{Problematização}

As unidades da APS são consideradas coordenadoras do cuidado e da comunicação com as demais redes do SUS e concretizam sua presença no espaço urbano por meio de sua localização em áreas limitadas denominadas "áreas de abrangência". Dois objetivos que integram as suas finalidades estabelecem relações com o espaço urbano de modo explícito: em primeiro lugar, elas devem oferecer atenção à saúde de forma completa; e, em segundo, devem favorecer as condições de integrar-se funcionalmente às outras unidades da rede. Quanto à primeira, as UBS cuidam hoje da promoção, prevenção 
e assistência à saúde coletiva, ultrapassando apenas a erradicação de doenças (Farias e Buchalla, 2005). Seu sucesso depende, portanto, da sua localização e das singularidades das comunidades onde se inserem, uma vez que a receptividade dos cidadãos é que as transformam em um elemento da vida urbana local. Ao se integrarem às outras unidades de saúde (Temporão, 2010), as UBS tendem a equilibrar a distribuição dos recursos necessários para uma atuação mais profícua, principalmente considerando que as densidades tecnológicas das redes de APS diferem entre si, e esse compartiIhamento evita a subutilização e o desperdício.

Dessa maneira, busca-se congregar uma relação horizontal, contínua e integrada em uma gestão compartilhada em consonância com as vizinhanças onde se localizam as UBS. A apropriação delas pela vizinhança parece ser capaz de proporcionar uma relação mais eficaz, promovendo o enraizamento da população e o uso espontâneo e participativo do ambiente urbano (Weil, 2001) integrado ao ato de residir. Simultaneamente, as UBS demandam uma integração territorial através dos fluxos de pessoas e de coisas, criando uma hinterlândia (zona de influência), que fortalece a rede e disponibiliza a tecnologia e os serviços.

A importância dos centros de saúde para as vizinhanças, considerando sua proximidade e inserção como enraizamento, talvez tenha sido registrada pela primeira vez quando do chamado Peckham experiment (Pearse e Crocker, 2013). Entre os anos de 1926 e 1950, Williamson e Pearse criaram o Pioneer Health Centre, na vizinhança de Peckham, sudeste de Londres (Figuras 1 e 2). Os objetivos dessa proposta pioneira e radical incluíam despertar nos moradores da região, população socialmente vulnerável, a valorização do cuidado à sua saúde por meio do uso rotineiro das instalações e dos serviços do centro, que incluíam piscinas, equipamentos para exercício físico e locais para a socialização. A proposta visava preservar a

Figuras 1 e 2 - Interior do edifício do Centro de Saúde de Peckham, Londres, hoje transformado em área de lazer privativa para um condomínio de apartamentos
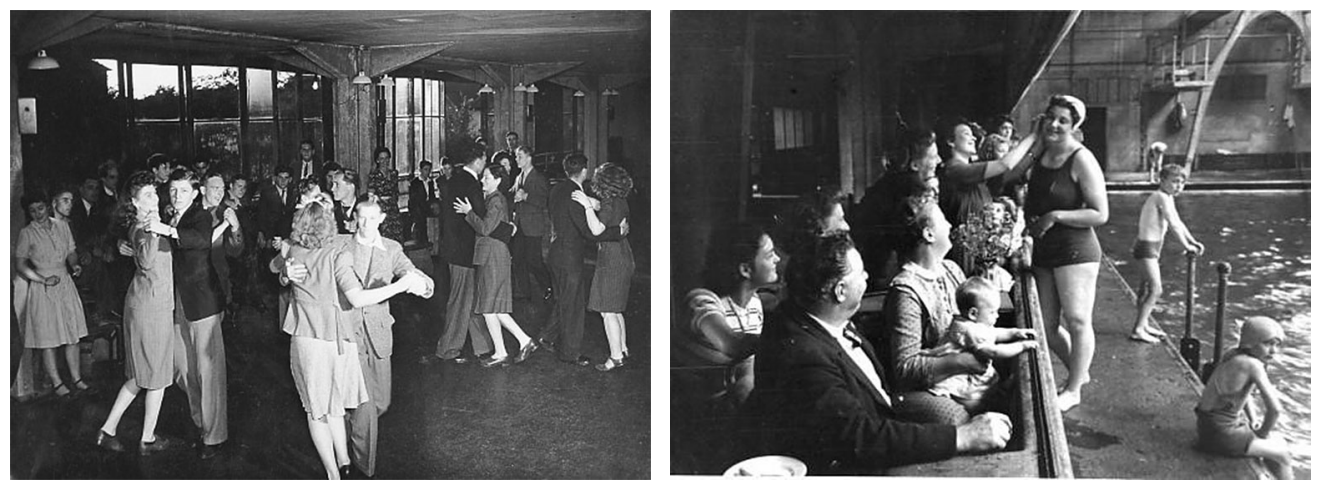

Fonte: Internet, acesso em 2016. 
assiduidade ao centro e, com ela, a prevenção, evitando o agravamento de diversas doenças.

Uma das primeiras reflexões teóricas sobre o conceito de espaço aplicado à saúde pública foi a de Snow, em Londres em 1854, por ocasião da epidemia de cólera. Desde então, 0 diferencial de saúde, conforme a localização no espaço urbano, vem sendo objeto de pesquisas (Silva, 1997) e, cada vez mais, as abordagens abrangem campos conjugados para a melhor compreensão dos fenômenos territoriais. Em se tratando da busca de uma totalidade entre o ambiente construído e a saúde das pessoas, Alexander e colegas (Alexander; Ishikawa e Silverstein, 1977, p. 236) argumentaram sobre a necessidade de se construírem as cidades em maior consonância com o ciclo vital e a idade das pessoas. Vizinhanças poderiam mesclar mais e melhor seus indivíduos e reconhecer os ritmos de passagem de um ciclo etário ao outro, com espaços mais apropriados às limitações individuais, como, por exemplo, a de movimentos. Aqueles autores também consideram que para um sistema de saúde que conseguir manter a saúde física e mental das pessoas deveria enfatizar ações relacionadas à manutenção da saúde e não somente ao tratamento de enfermidades. Consequentemente, o espaço deveria ser fisicamente descentralizado para se situar o mais próximo possível das atividades cotidianas, criando estímulos para práticas diárias que promovessem a saúde, tal como a simples sociabilidade, a natação, a dança, os esportes ao ar livre, além de tratamento médico incidental para essas atividades. Os autores terminam comentando que tais espaços sucumbem à percepção geral de que tratam somente de doenças e casos de enfermidades, e não de saúde.
Nos últimos 15 anos, a análise espacial vem sendo utilizada com ênfase na saúde para dar suporte ao conceito de risco, em função das múltiplas possibilidades advindas da correlação de variáveis como localização da população, recursos e fluxos através de sistemas de informação geográfica (GIS). A espacialização da situação de saúde de uma região urbana sobreposta, em seu mapeamento, às espacializações socioeconômicas, sanitárias e ambientais possibilita uma aproximação mais reveladora do que realmente acontece no espaço urbano, mostrando-se ser uma grande contribuição (Gondim et al., 2008). Entretanto, o uso desses procedimentos considera que o território ocupado por uma vizinhança, ao longo do tempo, confere-Ihe limites territoriais e físicos resultantes da acumulação de situações históricas, ambientais e sociais. A ênfase a essas variáveis do enraizamento, entretanto, dá-se na consideração de que tais fatores constituem informação sobre a capacidade de suas características ativarem condições particulares para a produção de doenças (Barcellos et al., 2002; Gondim et al., 2008).

Igualmente, para que se possa avaliar mais precisamente o impacto da localização dos serviços de atenção primária à saúde, é necessário o reconhecimento de tais territórios como delimitadores das unidades espaciais das vizinhanças. A extensão geométrica merece abarcar uma multiplicidade de dados para identificar mais e melhor o perfil demográfico, epidemiológico, administrativo, tecnológico, político, social e cultural que caracteriza seus habitantes e se expressa em um espaço urbano em permanente construção (Monken et al., 2008).

0 geógrafo brasileiro Milton Santos, considerado um dos grandes influenciadores 
do movimento que compreendeu o processo saúde-doença na cidade como manifestação social, atenta, em sua vasta bibliografia, para a necessidade de estudar tal processo através de questões espaciais e territoriais (Faria e Bortolozzi, 2009). Para auxiliar o estabelecimento de tais análises, nos últimos anos, possantes ferramentas estão disponíveis para investigar as correlações entre Eventos Relacionados à Saúde (ERS) e o espaço urbano.

Entretanto, apesar do crescimento exponencial do interesse pelo assunto em todo o mundo, a maioria das pesquisas continua "não espacial" (Auchincloss et al., 2012). Os dados espaciais são subutilizados assim como os instrumentos analíticos disponíveis. A definição de unidades espaciais tem gerado inúmeras bases, posto que várias instituições, ao pesquisarem os mesmos dados, criam novos recortes desconectados intersetorialmente e, por vezes, negligentes quanto à interoperabilidade. Esse descompasso compromete recursos financeiros, técnicos, pessoais e retarda a elaboração de políticas mais eficazes ou efetivas, negligenciando certas unidades não cobertas e, portanto, não atendidas.

No Brasil, o uso do ferramental contemporâneo também tem se revelado um campo em crescimento (Barcellos e Ramalho, 2002) cuja consolidação tem ocorrido com a disponibilização de dados de qualidade, a ampliação da capacidade computacional disponível e a capacitação profissional em GIS aplicado à Saúde. Um dos desafios contemporâneos é a pesquisa de novas possibilidades metodológicas para a compreensão da dinâmica urbana e o subsídio às ações de atenção à saúde (Perez, 2006), possibilitando uma base de dados dinâmica com coletas e consultas em tempo real.
Como mencionado, a definição da unidade espacial do território é ainda controversa nos estudos das condições de saúde no espaço urbano. Um dos principais desafios refere-se ao fato de que os dados dos ERS são coletados segundo a lógica territorial do SUS, que estabelece sua unidade espacial com objetivos administrativos. Essa lógica comanda a referência espacial na visualização dos dados sobre saúde no território, constituindo-se em uma imposição administrativa ao sistema privilegiado de informação, cujo custo pode mascarar singularidades das unidades.

Adotar unidades espaciais diferentes abre o caminho para a superação da invisibilidade de algumas correlações entre saúde e espaço. Este artigo se propõe a apresentar uma análise de um mesmo ERS, contido em distintos tipos de unidades espaciais, seja uma vizinhança identificável, seja uma bacia hidrográfica, seja um distrito sanitário, e que possa ser sobreposto aos dados demográficos, socioeconômicos, culturais e ambientais. Esse exercício permitirá visualizar a espacialização dos dados de acordo com o interesse do estudo, incorporando os preceitos de simultaneidade analítica e interação entre escalas diferentes.

\section{Estudo de caso}

A unidade de agregação mais adequada para avaliar o impacto de ações preventivas no espaço urbano parece ser a área delimitada ao redor dos Centros de Saúde (Barcellos e RamaIho, 2002) - sua hinterlândia, por critérios de abrangência bem caracterizados. A Secretaria Municipal de Saúde da cidade de Belo Horizonte (SMSA-BH) define as áreas de abrangência 
por homogeneidade socioeconômico-sanitária, isto é, áreas que reúnem espaços nos quais se concentram grupos populacionais internamente homogêneos, tendo em conta as "condições objetivas de existência".

Segundo a SMSA-BH,

[...] essas áreas permitem a condução da vigilância à saúde mediante ações interdisciplinares e intersetoriais conforme as características do grupo populacional residente. A microárea tem como unidades internas de análise e acompanhamento a moradia; espaço de vida de uma microunidade social, a família nuclear ou estendida. A moradia é o locus para o desencadeamento de intervenções de saúde, buscando a promoção do bem-estar da população. (Áreas de Abrangência dos Centros de Saúde / Gestão Compartilhada, s/d)

Este estudo de caso foi realizado, confrontando a unidade de abrangência definida pela SMSA-BH e uma unidade espacial calculada pela facilidade e importância no acesso peatonal e motorizado aos 147 UBS administrados pela SMSA-BH (Figura 3). Esses centros se encontram distribuídos em nove distritos sanitários e realizam mais de 2,5 miIhões de consultas médicas por ano. No caso de alguma necessidade de tratamento, informações ou cuidados básicos de saúde, a população deve se dirigir ao centro mais próximo de sua residência e, nele, agendar consultas rotineiras e/ou especializadas, fazer exames, acompanhar doenças crônicas, obter vacinas e medicamentos, dentre outros serviços. Cada UBS tem que cobrir uma área de abrangência definida, delimitada segundo a densidade populacional do distrito sanitário onde se situa. Essa área é atendida por equipes de profissionais de saúde, com base no tamanho de sua população e em critérios de vulnerabilidade de cada área (ibid.).

Entretanto, o conceito de abrangência é complexo, baseado no conceito de acesso ao serviço de saúde. Sua discussão, desde a década de 1950 (Travassos e Martins, 2004), constitui ainda hoje um campo fecundo de debate concernente à avaliação dos serviços de saúde. A dimensão tratada aqui é a técnica, relativa à planificação e à organização da rede de serviços, e merece ser incorporada às outras dimensões explicativas, tais como as da políti$\mathrm{ca}$, da economia e da cultura. A tentativa visa articular o referencial teórico e a definição conceitual aproveitando a diversidade de dados disponíveis sobre o tema em termos espaciais, escalares e temporais.

\section{Método}

Através da rede viária da cidade foram calculadas as rotas mais econômicas na delimitação das áreas de abrangência de cada UBS. Para sumarizar esse processo, foram detalhadas as etapas a seguir, com a indicação dos comandos para o aplicativo ArcGIS Pro, ${ }^{1}$ extensão Network Analyst. Tais comandos têm correspondentes bem similares em outros aplicativos, tais como o QGis e o Maplnfo, e o detalhamento neste artigo sumariza o método e permite sua adaptação para outras plataformas.

- Mapa das vias públicas

0 mapa com a linha central (Centerline map) representando a rede viária do município de Belo Horizonte foi obtido do site OpenStreet$\operatorname{Map}^{2}$ (OSM), do projeto colaborativo para a 


\section{Figura 3 - Localização dos centros de saúde da cidade de Belo Horizonte}

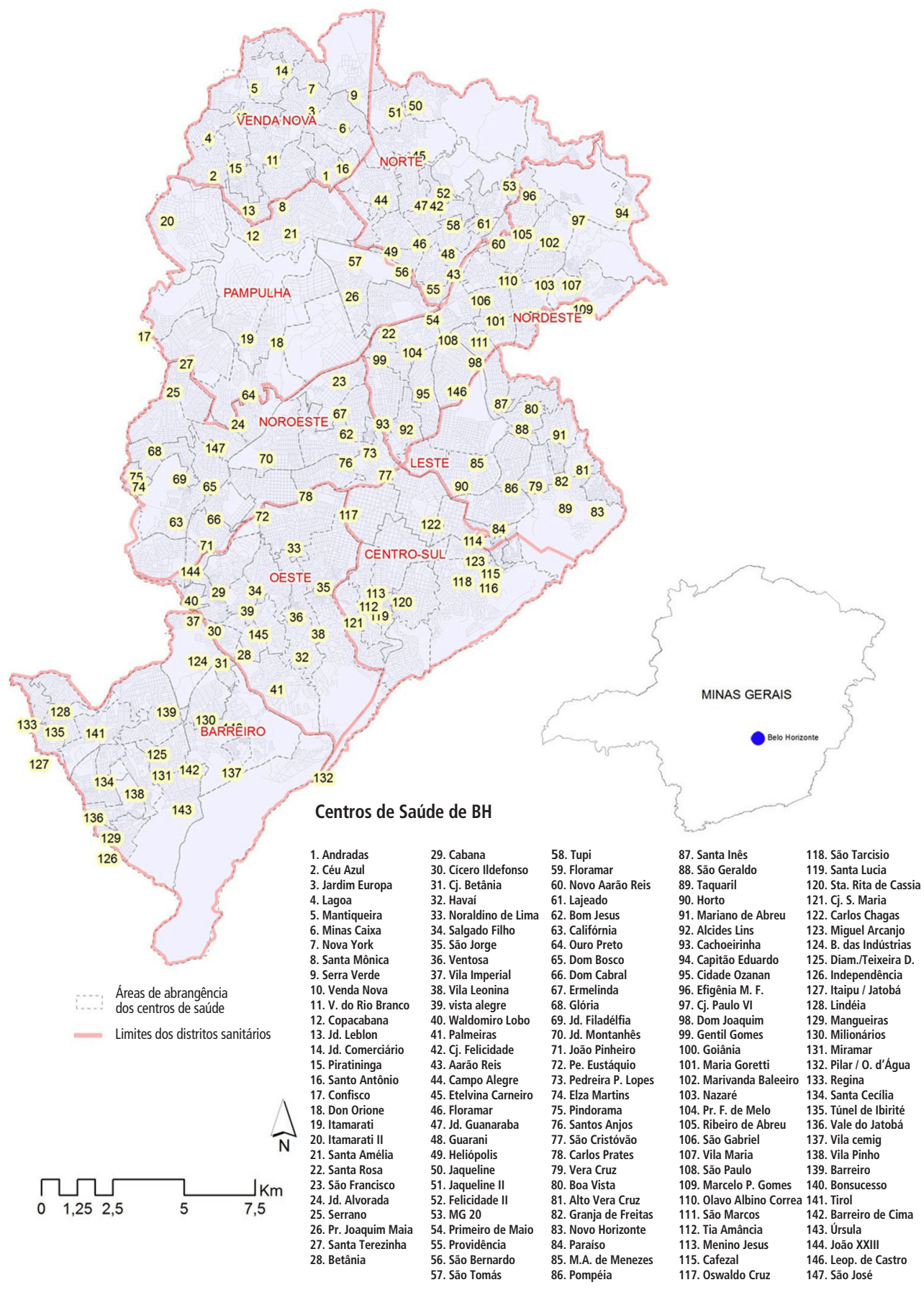

Fonte: os autores, com base em dados IBGE, 2010/PBH, 2014. 
criação de um mapeamento gratuito e editável de todos os espaços urbanos do mundo. A vantagem do material oferecido por esse site gratuito é que as informações disponibilizadas estão em contínua atualização pelos colaboradores, com qualidade compatível ao Network Analyst ARCmap.

- Composição do banco de dados para a análise da rede de vias públicas

A seguir, esse mapa recebeu algumas informações adicionais para compor uma base de dados para o Network Analyst. Foram indicados o tipo de cada segmento (ruas, rodovias, becos, etc.), a velocidade máxima permitida para os automóveis e a declividade do segmento viário. Para que o módulo do Network Analyst funcionasse corretamente, a rede foi dividida em segmentos conectados por vértices, que representam as esquinas e as demais junções (Figura 5).

Figura 4 - Todos os segmentos viários do Brasil

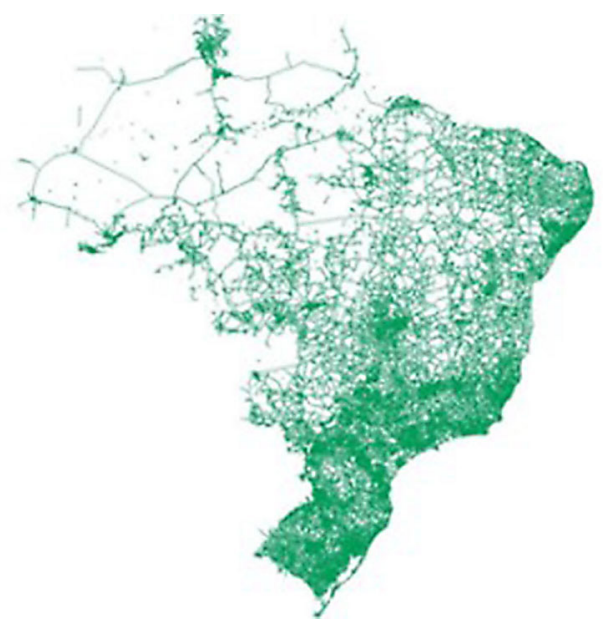

0 cálculo da declividade média de cada segmento das vias usou o modelo de cálculo mostrado na Figura 6, que é a vista do modelador matemático das operações, e pode ser compreendido por especialistas. 0 mapa da centerline já ajustado teve cada um dos 49.885 segmentos de via divididos em três pontos para garantir uma melhor estimação da declividade dos setores de via.

A seguir, utilizando o mapa com as feições das curvas de nível do município, foi criado um mapa clinográfico, e a declividade de cada ponto dos segmentos foi capturada numa nova tabela. A Figura 7 ilustra esse processo, apresentando uma camada espacial com a linha central dos segmentos de um trecho urbano. Produziu-se, com isso, um mapa das vias de todo o município de Belo Horizonte, contendo o valor da declividade para os três pontos, dos quais se calcularam a declividade média e o sentido aclive/declive.

Figura 5 - Split dos segmentos de vias

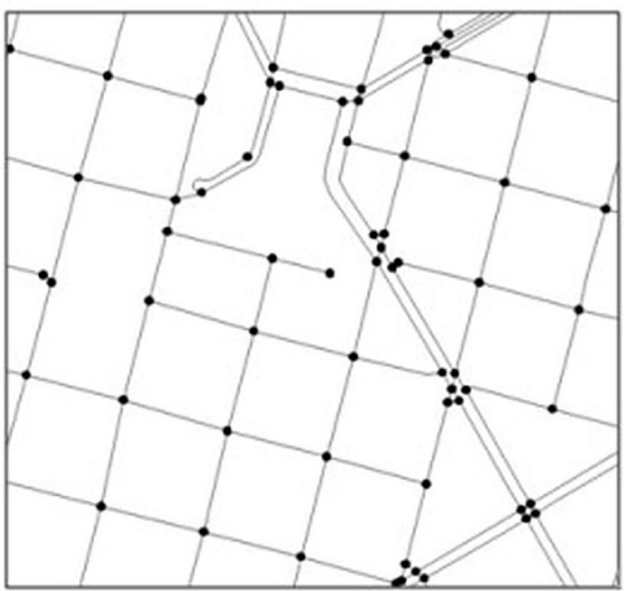

Fonte: os autores, com base em dados da OSM (2014). 
Figura 6 - Model Builder ArcGis para determinação da declividade média das vias

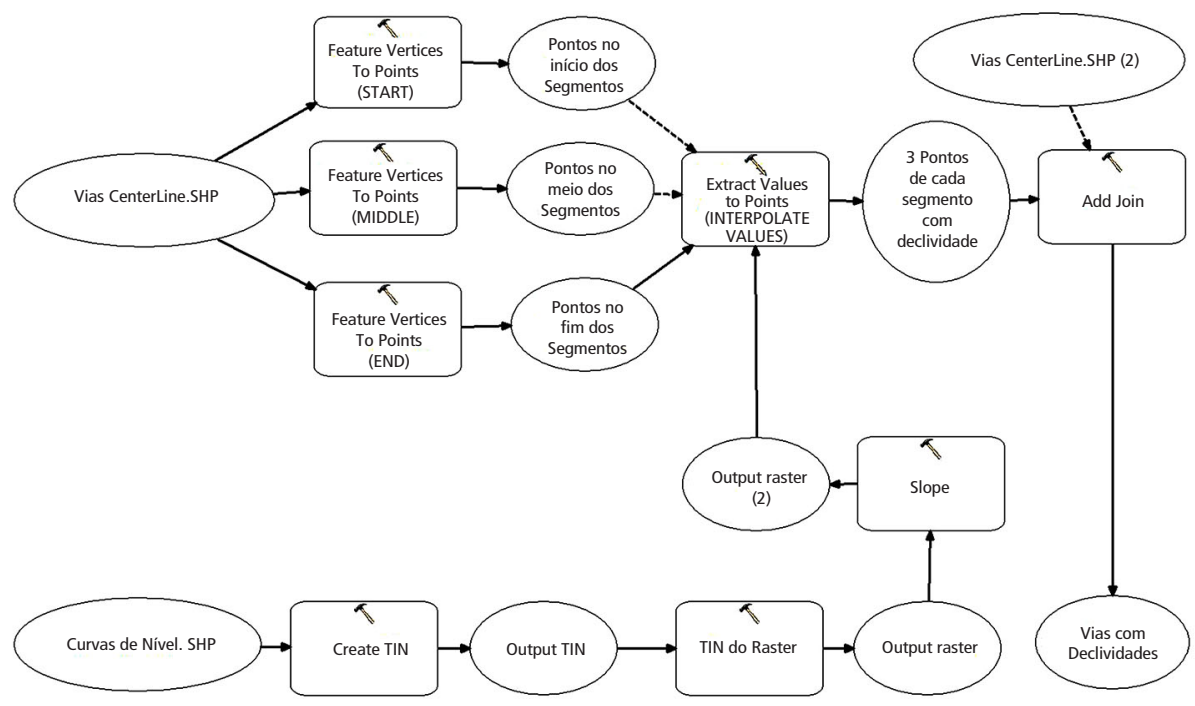

Fonte: os autores.

Figura 7 - Ilustração das camadas sobrepostas para identificação de dados para os segmentos viários

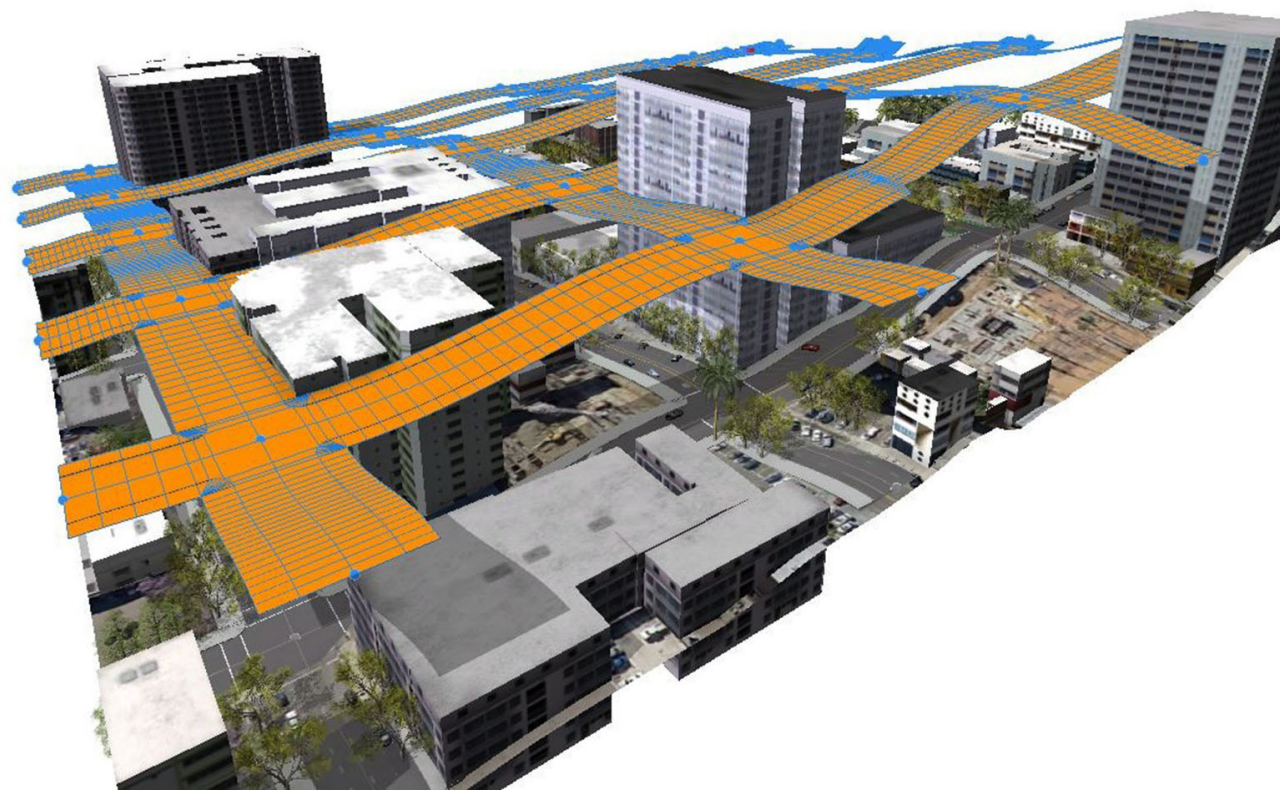

Fonte: os autores. 
- Implementação dos dados de impedância A análise de redes viárias pode considerar pedestres e veículos. Para o primeiro caso, leva em conta a velocidade média da caminhada das pessoas, assim como a variação de velocidades conforme a declividade das vias, 0 sentido da caminhada (se subida ou descida) e a idade do pedestre. Para o segundo caso, consideram-se as informações sobre a hierarquia das vias, o volume e o sentido do tráfego, o sentido das conversões e as velocidades máximas permitidas.

No nosso caso, o movimento peatonal foi prioritário, devido às características da avaliação do acesso às UBS pelos moradores. Já as condições de facilidade de movimentação em função dos elementos da logística e do princípio de compartilhamento de recursos tecnológicos e de serviços especializados foram avaliadas para os valores do cálculo de tráfego motorizado. A seguir, sumarizam-se os elementos presentes no cálculo desses dois tipos de movimentos.

A resistência oferecida aos pedestres e aos carros em seus trajetos é denominada "impedância" nos estudos de GIS. Conforme se mencionou, devem ser considerados valores médios para as velocidades das caminhadas sob influências diversas, desde a declividade e idade dos pedestres, até a temperatura e densidade de pessoas nos passeios. Existem hoje muitas pesquisas dirigidas ao assunto (Daamen; Hoogendoorn e Bovy, 2005; Hoogendoorn e Bovy, 2002; Hoogendoorn; Bovy e Daamen, 2002). Aqui priorizamos o Gráfico 1 , que relaciona a declividade em $\%(x)$ e a velocidade da caminhada em metros por minuto $(\mathrm{y})$. Os valores negativos referem-se à declividade em sentido descendente e os positivos, em sentido ascendente (Bovy, 1973).

Gráfico 1 - Velocidade e declividade no movimento de pedestres

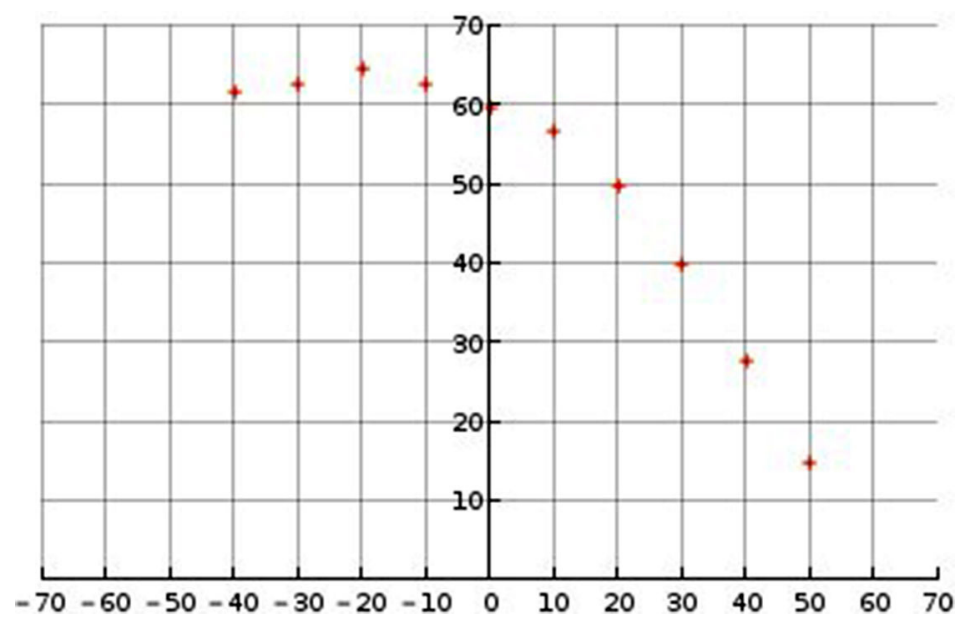

Fonte: Bovy (1973). 
Já, para o estudo de impedância dos automóveis, a tabela dos segmentos viários recebeu dados que armazenam a informação acerca do sentido das vias para circulação dos veículos, declividade e sentido, largura da via, velocidade e tipologia. Todos esses dados, como se disse anteriormente, foram retirados do OSM, mas vale comentar que o software ArcGIS 10.14 (2016) já permite a conexão do aplicativo em tempo real com os bancos de dados atualizados pelas instituições responsáveis pelo controle do trânsito, como acontece em cidades como Londres e Paris, onde até o volume de tráfego é disponibilizado nos sites das prefeituras. Entretanto, as informações sobre 0 sentido das vias foram suficientes para finalizar o conjunto de dados do mapa de segmentos de vias e possibilitou avançar com a análise de rede através do aplicativo ArcGIS. Aquele software utiliza algoritmos matemáticos disponíveis no módulo de geoprocessamento Network Analyst, ${ }_{1}^{3}$ os quais são baseados no algoritmo clássico de Dijkstra (Skiena, 1990) que permite o cálculo da rota de menor impedância (custo, esforço) entre um ponto e outro, no interior de uma rede - o que pode gerar uma análise intrassetorial. Limitamo-nos a indicar a existência de uma vasta bibliografia sobre o algoritmo de Dijkstra, que pode servir aos interessados. Tal algoritmo foi aplicado a um grande número de problemas a partir de 1956, quando foi concebido e se revelou uma formulação lógica e inovadora em diversos campos. No desenho urbano, ele tornou possível várias análises sobre a acessibilidade, sendo incorporado em aplicativos contemporâneos (Steinitz, 2012), como o ArcGis.

A etapa final consistiu na criação de duas redes, a partir da camada das vias implementadas com as informações anteriores, uma para análise dos movimentos de automóveis e outra para o movimento de pedestres. Foi necessária a programação de um roteiro na linguagem Python ${ }^{4}$ para reconhecer a sinalização de sentido das ruas da cidade, no caso da análise da rede para automóveis. Igualmente, a rede para análise do movimento de pedestres demandou um roteiro associando os dados de velocidade e a declividade dos passeios, como anteriormente dito. A inserção desses roteiros foi feita na etapa do que se chama DataSetNetwork, ilustrado na Figura 9.

Figura 8 - Atributos para a avaliação das regras do modelo matemático construído para os automóveis

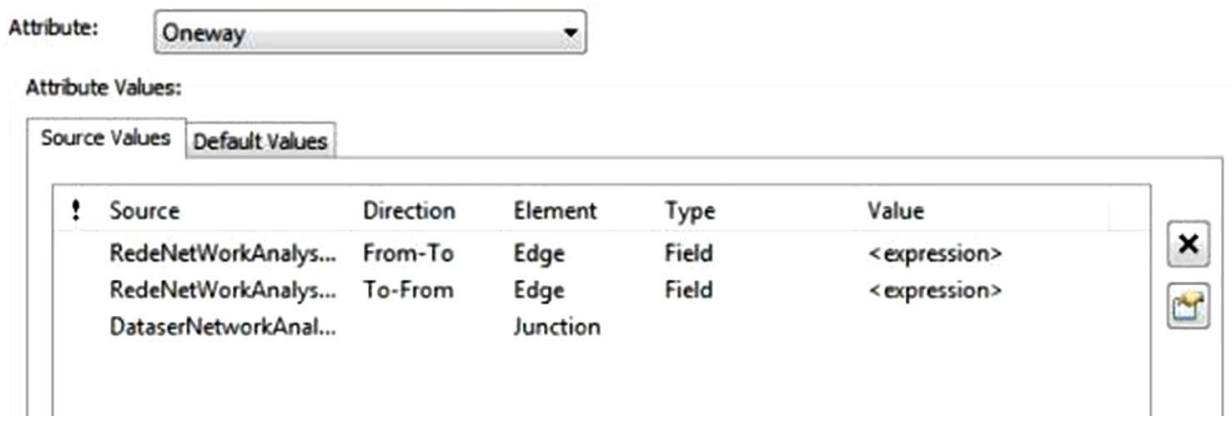


Esses scripts regulam a velocidade dos pedestres e calculam o tempo que eles gastariam para percorrer os segmentos de passeio. A velocidade é determinada segundo a declividade do trecho, de acordo com o Gráfico 1. A partir disso, avaliaram-se os sentidos dos movimentos dos pedestres, se subida (aclive) ou se descida (declive propriamente dito).

\section{Resultados e análises}

0 conjunto de dados trabalhados e relatados para a construção da base nos facultou descrever alguns dos resultados para melhor compreender a influência peatonal, a influência de motorizado e a análise da localização-distribuição. Então, vejamos.

- Influência Peatonal (Service Area) ${ }^{5}$ Nesta análise foram considerados os dados de pedestres e de automóveis para observar o comportamento no acesso às USB. A Figura 9 mostra o cálculo da área de abrangência das UBS, com tempos de caminhadas dos pedestres de até 15 minutos. Ressalte-se que os parâmetros para esse cálculo foram as velocidades compreendidas em acordo com a declividade, como explicitado anteriormente. Dessa maneira, a Figura 9 mostra um acesso bastante reduzido pelo trajeto a pé, segundo os parâmetros

Figuras 9 e 10 - Service Area com impedâncias de velocidade peatonal, considerando aclive e declive
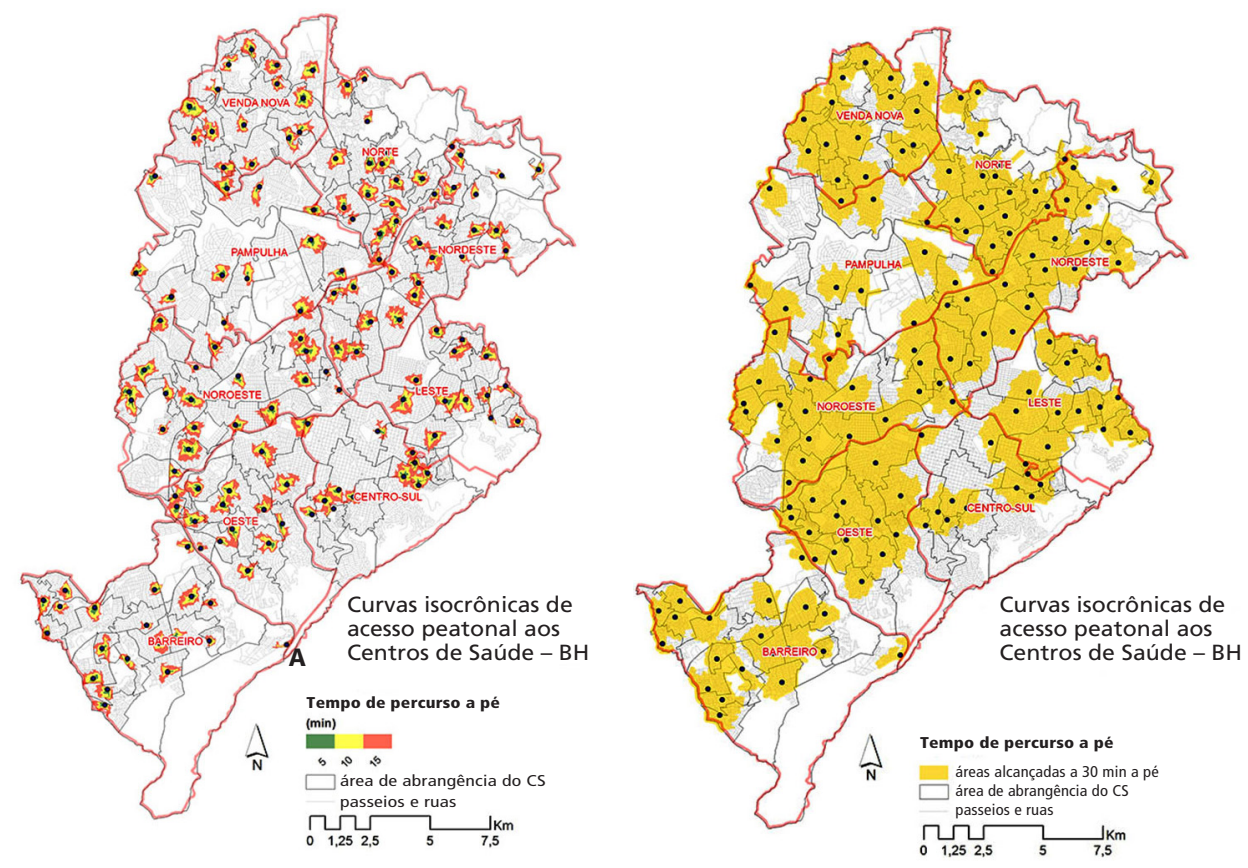

Fonte: os autores. 
aqui adotados. Essa situação fica ainda mais crítica nas UBS da Regional Barreiro, que possuem as menores áreas sob as curvas isocrônicas. Já o resultado mostrado no mapa da Figura 10 considera o tempo de caminhada de $30 \mathrm{mi}$ nutos. A comparação desses dois mapeamentos indica uma redução da área de influência da UBS e, simultaneamente, pouca correspondência em relação às áreas de abrangência determinadas pelo SUS/PBH. Também dela pode-se concluir a necessidade de pesquisar conceitos relativos às "distâncias caminháveis" na cidade - walking distance, walkability (Leslie et al., 2007; Murekatete e Bizimana, s/d). Tais conceitos possuem muitas referências na literatura sobre saúde orientadas para doenças cardíacas e respiratórias. Entretanto, há poucos dados sobre o que ocorre no Brasil.

A junção dos polígonos da Figura 10 sugere a existência de anéis isocrônicos que comunicam as UBS em caminhadas de $30 \mathrm{mi}-$ nutos. $A$ área dos anéis pode se constituir num indicador, mas ele tem pouca utilidade, uma vez que as curvas são calculadas pelo tempo de percurso nas ruas e passeios. Algumas UBS do Barreiro, como por exemplo a UBS Olhos D'água (marcada como A na Figura 9), tem a menor área de influência, caracterizada pela interferência da declividade. Finalmente, outra reflexão possível se refere à caracterização dos pedestres por idade que, numa pesquisa futura, poderá indicar o estabelecimento de

Figura 11 - Service Area de motorizados

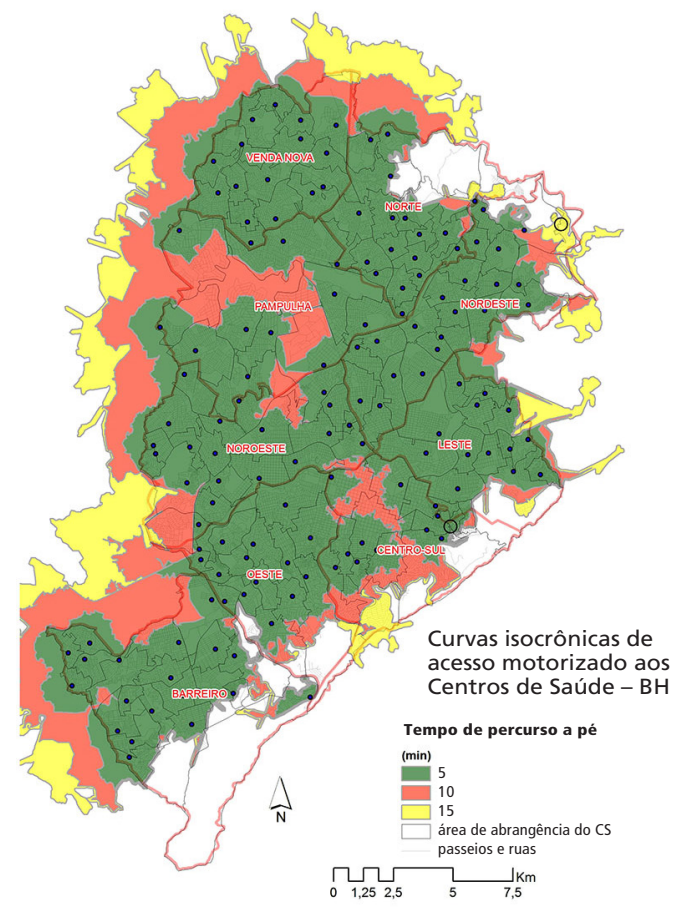

Fonte: os autores. 
velocidades diferentes para a abordagem. No caso em estudo, o padrão de velocidade foi generalizado para ser inclusivo, considerando pessoas acima de 65 anos.

- Influência de motorizado (Service Area) ${ }^{6}$ A Figura 11 exibe o cálculo das áreas de influência das UBS acessadas por automóveis. Um problema relacionado ao estágio tecnológico do cenário brasileiro é a falta de dados sobre otráfego de automóveis e pedestres. 0 volume do tráfego e a densidade peatonal são dados empíricos que necessitam de medição atualizada, frequente e livre, durante todos os dias do ano, para que seja possível alcançar alguma preditibilidade. No caso, foram consideradas as velocidades máximas dos segmentos de vias, o que não é um estado ideal e, de modo algum, preciso. Tal precariedade indica que os instrumentos baseados na impedância temporal irão falhar nesse contexto, inviabilizando a utilização de algumas ferramentas do Network Analyst ArcGIS, que tem potencial, como se mencionou anteriormente, de conectar-se à base de dados em tempo real das instituições reguladoras do trânsito.

- Localização-Distribuição (Location-Allocation)

Esse tipo de teste permite visualizar as áreas ótimas de demanda a serem atendidas pela UBS. Essa análise resulta num conjunto de linhas que sintetiza o trajeto pelos segmentos de vias desde a UBS até o ponto de demanda, ou seja, pontos de endereços das edificações que possuem a menor impedância de tempo. A Figura 12 estabelece retas que vão de cada edifício da Regional Barreiro até a UBS mais econômica (menor impedância de tempo a pé). Diferindo da análise de Service Area, este estudo possibilita identificar e listar quais os números de domicílios e outros que estão sobre a influência da UBS. Se for considerado um indicador, será possível determinar quantas conexões com domicílios serão suportadas para a capacidade de cada UBS em intervalos temporais.

A exemplo de sobreposição de outros dados, a Figura 13 apresenta o Índice de Vulnerabilidade à Saúde (IVS) e a população com mais de 65 anos em cada setor censitário. 0 polígono continente de todas as retas de tempo poderia ser o agregador dos dados da população idosa, contribuindo para relocalizar setores censitários de forma distinta, produzindo áreas de agregação com indicadores que possibilitassem maior homogeneidade espacial. Esta última observação expressa a necessidade de análises que favoreçam a reconstrução de novas unidades de agregação significativas, concomitantemente com uma mudança conceitual das UBS na sua relação com as vizinhanças.

\section{Conclusões}

Este estudo de caso pretendeu demonstrar a potencialidade de investigações sobre o território e a saúde, no que tange aos possíveis condicionantes espaciais de maior ou menor acesso às UBS em um contexto urbano de vivência, acenando para o enriquecimento do debate sobre os rumos da saúde no Brasil.

Preliminarmente, o estudo verificou 0 conceito do centro de saúde de modo positivo e integrado à vizinhança. A seguir, expôs, de acordo com os parâmetros aqui adotados, um acesso mais reduzido pelo trajeto a pé, além de descrever algumas UBS com restritas áreas de 
Figura 12 - Location-Allocation com menor impedância de velocidade peatonal considerando aclive e declive

Tempo de caminhada origem nas residências e destino Centro de Saúde Regional Barreiro

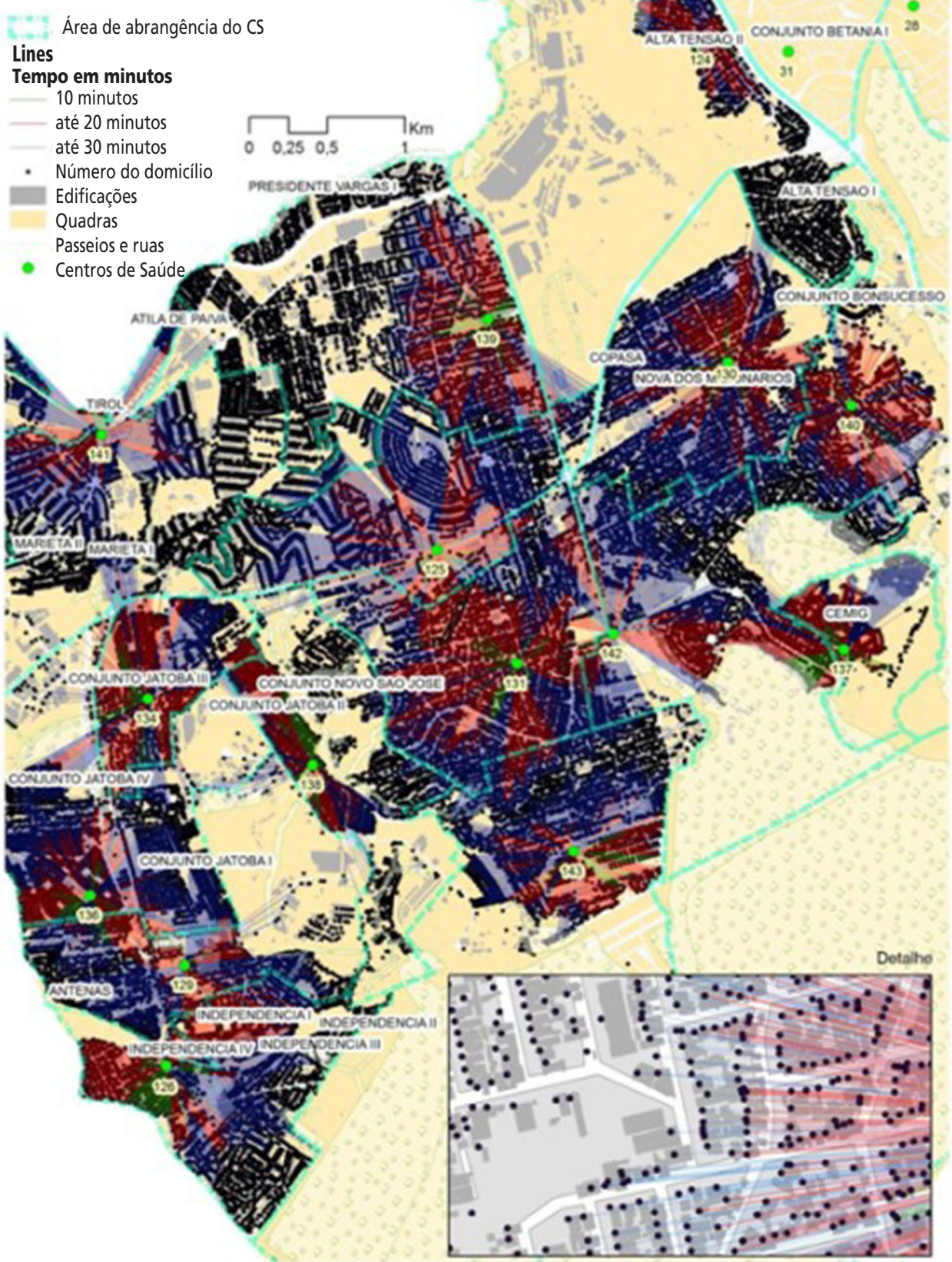


Figura 13 - Location-Allocation sobreposições de dados: população idosa e Índice de Vulnerabilidade à Saúde (IVS)

Tempo de caminhada origem nas residências e destino Centro de Saúde Regional Barreiro 2.ent. Área de abrangência do CS Tempo de caminhada aos CS's

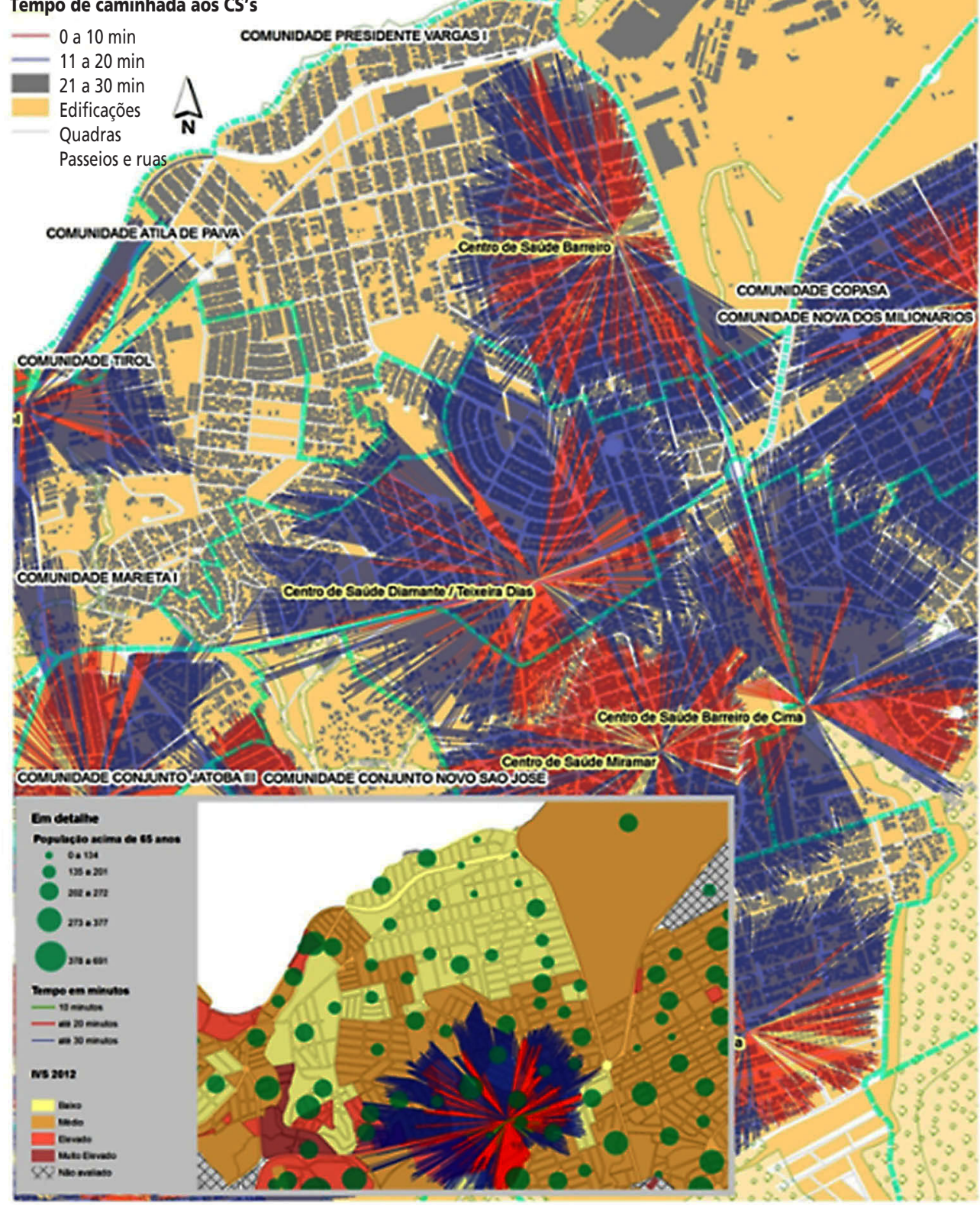

Fonte: os autores. 
abrangência, sugerindo uma pequena área de influência e ínfima correspondência com a respectiva área administrativa. Essa ausência de sobreposição tende a sugerir limitações entre as áreas (de influência e administrativa), o que compromete a eficácia de políticas planejadas para um recorte determinado. Tal descompasso possivelmente oculta singularidades de áreas mais próximas ao que, de fato, ocorre na realidade, em termos de obstáculos a uma redução no tempo de deslocamento e de prestação do serviço. 0 estudo dos obstáculos do território é assunto que carece de pesquisa longa e pode ser tratado com as mesmas bases operacionais aqui descritas, detendo-se em verificações importantes como modelos de percepção ambiental, cognição e geografia (Wood; Kitchin; Blades, 2002) e saúde.
0 artigo permite concluir sobre a necessidade de maior multiplicidade de informações de qualidade para o estudo de indicadores de Saúde Urbana através de ferramental analítico utilizando o GIS. Recomenda-se, também, a realização de pesquisas sobre conceitos relacionados às "distâncias caminháveis" e os impedimentos ou barreiras constantes nos percursos, com a imagem da cidade (Lynch, 1997).

As reflexões aqui aquecem o debate no que se refere às diversas definições e possibilidades analíticas do que é um contexto urbano, seja ele originado de um ponto de vista objetivo, como os setores administrativos, seja ele de origem subjetiva, tais como a percepção/convivência da vizinhança pela população adstrita a um determinado território urbano.

\section{Renato Cesar Ferreira de Souza}

Universidade Federal de Minas Gerais, Escola de Arquitetura, Departamento de Projetos, Núcleo de Pós-Graduação em Arquitetura e Urbanismo. Belo Horizonte, MG/Brasil.

rcesarfs@gmail.com

\section{Veneza Berenice de Oliveira}

Universidade Federal de Minas Gerais, Escola de Medicina, Programa de Pós-Graduação em Saúde Pública. Belo Horizonte, MG/Brasil.

veneza@medicina.ufmg.br

\section{Doralice Barros Pereira}

Universidade Federal de Minas Gerais, Departamento de Geografia, Programa de Pós-Graduação em Geografia. Belo Horizonte, MG/Brasil.

pereiradb@yahoo.com.br

\section{Heloisa Soares de Moura Costa}

Universidade Federal de Minas Gerais, Departamento de Geografia, Programa de Pós-Graduação em Geografia. Belo Horizonte, MG/Brasil.

heloisasmcosta@gmail.com

\section{Waleska Teixeira Caiaffa}

Universidade Federal de Minas Gerais, Escola de Medicina, Programa de Pós-Graduação em Saúde Pública. Belo Horizonte, MG/Brasil.

caiaffa.waleska@gmail.com 


\section{Notas}

(1) "ArcGIS Platform - Esri", 2005. Disponível em <http://www.esri.com/software/arcgis>. Acesso em: 26 dez 2015.

(2) "OpenStreetMap Foundation Wiki", 2015. Disponível em: <https://wiki.osmfoundation.org/>. Acesso em: 4 jan 2016.

(3) "ArcGIS Platform - Esri", 2005. Disponível em: <http://www.esri.com/software/arcgis>. Acesso em: 26 dez 2015.

(4) "Welcome to Python.org", 2004. Disponível em <https://www.python.org/>. Acesso em: $20 \mathrm{abr}$ 2016.

(5) Service Area pode ser entendida como a área de influência de um determinado serviço ou edifício. Essa análise considera a impedância para os tempos e deslocamentos de pedestres, conforme explicado.

(6) Considerando a impedância para automóveis, como se explicou.

\section{Referências}

ALEXANDER, C.; ISHIKAWA, S. e SILVERSTEIN, M. (1977). A pattern language: towns, buildings, construction. Nova York, Oxford University Press.

Áreas de Abrangência dos Centros de Saúde / Gestão Compartilhada (s/d). Disponível em: http:// gestaocompartilhada.pbh.gov.br/estrutura-territorial/areas-de-abrangencia-dos-centros-desaude. Acesso em: 4 jan 2016.

AUCHINCLOSS, A. H. et al. (2012). A Review of Spatial Methods in Epidemiology, 2000-2010. Annual review of public health, v. 33, n. 1, pp. 107-122.

BARCELLOS, C. de C. et al. (2002). Organização espacial, saúde e qualidade de vida: análise espacial e uso de indicadores na avaliação de situações de saúde. Informe Epidemiológico do Sus, v. 11, n. 3, pp. 129-138.

BARCELLOS, C. DE C. e RAMALHO, W. M. (2002). Situação atual do geoprocessamento e da análise de dados espaciais em saúde no Brasil. Informática pública, v. 4, n. 2, pp. 221-230.

BOVY, P. (1973). Reseaux et Espaces Piétonniers. s.I., Institut de Technique des Transports.

DAAMEN, W.; HOOGENDOORN, S. e BOVY, P. (2005). First-Order Pedestrian Traffic Flow Theory. Transportation Research Record: Journal of the Transportation Research Board, v. 1934, pp. 43-52.

Decreto n. 7508. Disponível em: <http://www.planalto.gov.br/ccivil_03/_ato2011-2014/2011/ decreto/D7508.htm>. Acesso em: 2 jan 2016. 
FARIA, R. M. e BORTOLOZZI, A. (2009). Espaço, território e saúde: contribuições de Milton Santos para o tema da geografia da saúde no Brasil. s.I., Editora UFPR. Disponível em: <http://www.rets.epsjv. fiocruz.br/sites/default/files/espaco_territoiro_e_saude.pdf>.

FARIAS, N. e BUCHALLA, C. M. (2005). The international classification of functioning, disability and health: concepts, uses and perspectives. Revista brasileira de epidemiologia - Brazilian journal of epidemiology, v. 8, n. 2, pp. 187-193.

GONDIM, G. M. de M. et al. (2008). “O território da saúde: a organização do sistema de saúde e a territorialização". In: MIRANDA, A. C.; BARCELLOS, C.; MOREIRA, J. e MONKEN, M. (orgs.). Território, ambiente e saúde. Rio de Janeiro, Fiocruz.

HOOGENDOORN, S. e BOVY, P. (2002). Normative pedestrian behaviour theory and modelling. Transportation and traffic theory.

HOOGENDOORN, S. P.; BOVY, P. e DAAMEN, W. (2002). Microscopic pedestrian wayfinding and dynamics modelling. Pedestrian and evacuation dynamics, v. 123, p. 154.

LESLIE, E. et al. (2007). Walkability of local communities: using geographic information systems to objectively assess relevant environmental attributes. Health \& place, v. 13, n. 1, p. 111-122.

LYNCH, K. (1997). A imagem da cidade. São Paulo, Martins.

MONKEN, M. et al. (2008). “O território na saúde: construindo referências para análises em saúde e ambiente". In: MIRANDA, A. C.; BARCELLOS, C; MOREIRA, J. C. e MONKEN, M. (orgs.). Território, ambiente e saúde. Rio de Janeiro, Fiocruz.

MUREKATETE, R. M. e BIZIMANA, J. P. (s/d). A GIS-based Approach for Developing Urban Walkability Indices: The Case of Kigali City, Rwanda.

ORGANIZAÇÃO MUNDIAL DA SAÚDE (2008). Relatório Mundial de Saúde 2008 - Cuidados de Saúde Primários, Agora Mais Que Nunca. s.l., World Health Organization.

PEARSE, I. H. e CROCKER, L. H. (2013). The Peckham Experiment: a study of the living structure of society. Edimburgo, Routledge.

PEREZ, G. (2006). Adoção de inovações tecnológicas: Um estudo sobre o uso de sistemas de informação na área de saúde. São Paulo, Universidade de São Paulo.

PITCHON, A. (2013). Índice de Vulnerabilidade da Saúde 2012. Belo Horizonte, Prefeitura de Belo Horizonte.

SILVA, L. J. da (2997). The concept of space in infectious disease epidemiology. Cadernos de Saúde Pública, v. 13, n. 4, pp. 585-593.

SKIENA, S. (1990). Dijkstra's Algorithm. Implementing Discrete Mathematics: Combinatorics and Graph Theory with Mathematica, Reading, MA: Addison-Wesley, pp. 225-227.

STEINITZ, C. (2012). A Framework for Geodesign: Changing Geography by Design. s.I., Esri Press.

TEMPorÃo, J. G. (2010). Portaria no 4.279, de 30 de dezembro de 2010. Disponível em: <http:// conselho.saude.gov.br/ultimas_noticias/2011/img/07_jan_portaria4279_301210.pdf>. Acesso em: 1 mar 2016.

TRAVASSOS, C. e MARTINS, M. (2004). Uma revisão sobre os conceitos de acesso e utilização de serviços de saúde. Cadernos de Saúde Pública, v. 20. 
WEIL, S. (2001). O enraizamento. Bauru, Editora da Universidade do Sagrado Coração.

WOOD, D.; KITCHIN, R. e BLADES, M. (2002). The cognition of geographic space. s.l., I. B. Tauris \& Co.

Texto recebido em 14/jan/2016

Texto aprovado em 31/mar/2016 